\title{
Oriented Lamellar Structure and Pore Formation Mechanism in CSX-Processed Porous High-Density Polyethylene
}

\author{
Shujun Chen, ${ }^{\dagger}$ Souvik Nandi, ${ }^{*}$ H. Henning Winter, ${ }^{*, *}$ and Samuel P. Gido ${ }^{*, \dagger}$ \\ Department of Polymer Science and Engineering and Department of Chemical Engineering, \\ University of Massachusetts Amherst, Amherst, Massachusetts 01003
}

Received May 27, 2005; Revised Manuscript Received January 13, 2006

\begin{abstract}
Characterization of pore structure and pore wall crystal structure was performed on porous highdensity polyethylene (HDPE) material using scanning electron microscopy (SEM), transmission electron microscopy (TEM), and electron diffraction (ED). The porous HDPE material was obtained through crystallization from swollen cross-linked polyethylene gels (CSX process $\left.{ }^{1}\right)$ in supercritical propane. SEM showed an open-pore structure of micron-sized pores, large void fraction, and surface area as well as thin yet rigid pore walls, making this material a good candidate for a variety of applications. TEM revealed oriented lamellar structure in the pore walls which was much different from structures found in typical bulk HDPE as well as that of the cross-linked HDPE before CSX processing. Electron diffraction results confirmed the presence of oriented lamellar stacking. On the basis of this oriented lamellar structure, possible mechanisms for crystallization and pore formation are suggested.
\end{abstract}

\section{Introduction}

Porous polymeric materials are used in a large variety of applications, such as catalysis, separations, sound absorption, thermal and electrical insulation, ${ }^{2-5}$ and biomedical applications. ${ }^{6-10}$ Typical techniques to produce porous materials include foaming, leaching, sintering, extrusion, injection molding, polymer precipitation, and thermally induced phase separation. ${ }^{11,12}$ However, these processes in general do not offer optimal control over pore structure (pore size, geometry, and interconnectivity) and bulk characteristics (density, void fraction, mechanical and electrical properties). To address this issue, several new processes ${ }^{1,13-15}$ have been developed in recent years. Shastri, Martin, and Langer ${ }^{13}$ developed a hydrocarbon templating process for the production of macroporous polymer foams, in which a hydrocarbon particulate phase is used as a template for the precipitation of the polymer phase and subsequent pore formation. Polymer foams obtained through this method have large pores $(>100 \mu \mathrm{m})$ nearly identical to the particulate hydrocarbon phase employed, in terms of both geometry and size. Ko and co-workers ${ }^{14}$ utilized electrospinning to create an interconnecting pore structure formed by electrospun poly(DLlactide-co-glycolide) (PLGA) nanofibers with diameters ranging from 500 to $800 \mathrm{~nm}$. The porous PLGA material thus created has a broad pore diameter distribution, high porosity, and mechanical properties that are well-suited for tissue-engineering scaffold applications. More recently, Sakai and co-workers ${ }^{15}$ reported the fabrication of a poly(L-lactic acid) (PLLA) scaffold possessing a 3D flow channel network through repeated layering/micromachining of macroporous sheets of PLLA/ $\mathrm{NH}_{4} \mathrm{HCO}_{3}$ salt particle composite followed by salt leaching and gas forming.

Among these new processes, crystallization of polymers from swollen cross-linked gels $(\mathrm{CSX})^{1}$ is one that offers a good combination of controllability, material properties, and environmentally friendliness. The CSX process uses a supercritical

\footnotetext{
$\dagger$ Department of Polymer Science and Engineering.

$\doteqdot$ Department of Chemical Engineering.

* To whom correspondence should be addressed. E-mail: gido@ mail.pse.umass.edu or winter@ecs.umass.edu.
}

fluid (SCF) to swell a cross-linked network of a crystallizable polymer above its melting temperature. This is followed by cooling and crystallization of the polymer in the SCF. Venting of the SCF above the critical temperature of the fluid but below the melting temperature of the polymer leaves behind a rigid open-pore structure. The SCF can be cleaned and recycled into the CSX process, which makes it an environmentally friendly zero-emission process. Porous materials made through CSX have open-pore structures with sterile surfaces, large specific surface areas, and controllable pore sizes and overall shapes, making them ideal candidates for biomedical applications. The fact that such low-cost thermoplastics as polyethylene and polypropylene can be easily made into well-controlled porous materials through CSX makes this process even more attractive.

Since the discovery of this novel process, attempts have been made in trying to understand the mechanism of pore formation during CSX. It was previously suggested by Nandi, Winter, and Fritz $^{16}$ that the pore structure forms in the following way: Preshaped and cross-linked polymers are swollen in a supercritical fluid above the crystal melting temperature of the polymer. The polymer in the swollen gel state is amorphous. Upon cooling below the crystallization temperature while maintaining pressure, fractions of the polymer crystallize in a nucleation and growth process and phase-separate from the fluid. Large composition fluctuations develop due to the crystallization process. Alternatively, some of the polymer may first separate from the fluid and then crystallize. In either case, the result is a bicontinuous morphology in which one phase is polymer-rich (crystalline polymer and polymer-rich amorphous phase) and the other is mostly fluid. The swelling fluid creates a continuous phase, which after its release leaves behind an open-pore structure. In addition, the cross-linking was assumed to play an important role in the phase separation process by suppressing large-scale polymer diffusion during crystallization.

Such a hypothesis paints a general picture of the pore formation process, but the detailed mechanism remains unexplained. For example, it is unknown whether the gel fraction (cross-linked segments) or the sol fraction (loose chains) nucleates first in the crystallization process. It is also unclear 
whether there is liquid-liquid phase separation before the onset of crystallization. We feel that a clear understanding of the polymer crystal morphology within the pore walls will help us better understand the pore formation process in CSX.

Direct observation of the pore wall morphology in TEM is thus desired, since TEM together with electron diffraction should allow determination of the crystal orientation inside the pore wall if such orientation exists. However, the porous nature of the material makes it difficult to obtain suitable TEM samples. In fact, to our knowledge, no previous work has been done on the internal structural characterization of pore walls in porous crystalline polymers using direct observation techniques such as TEM, mainly due to the difficulty in obtaining suitable samples without disrupting the structure. To overcome this challenge, special care was taken in cryo-microtoming to obtain thick sections of the porous sample. It is conceivable that this technique may also be applied to TEM sample preparation of porous materials made through other processes.

The aim of this paper is to study the pore wall crystal morphology and in turn the pore formation mechanism in the CSX process. High-density polyethylene (HDPE), the simplest and most studied crystalline polymer, is chosen as our model system. The overall pore structure of CSX-processed HDPE is characterized by SEM. The crystal morphology of HDPE before CSX and the pore wall morphology of HDPE after CSX are studied using TEM and electron diffraction. On the basis of the pore structure and the pore wall morphology, crystallization and pore formation mechanisms in the CSX process are proposed.

\section{Experimental Section}

Materials. The cross-linked high-density polyethylene (HDPE10) used in this study was industrial grade HDPE in the form of solid sheets. The method of cross-linking employed was e-beam at an irradiation dose of $100 \mathrm{kGy}$.

Gel Fraction Measurement. The gel fraction $\phi_{\text {gel }}$ of the crosslinked HDPE (weight fraction of connected network with respect to the whole sample) was determined using an ASTM method. ${ }^{16}$ A preweighed sample of mass $m_{0}$ was exposed to $p$-xylene for 72 $\mathrm{h}$ at $110^{\circ} \mathrm{C}$, the solvent being replenished every $24 \mathrm{~h}$. This extracted most of the loose chains ( undissolved in the vessel. It was recovered and dried for $24 \mathrm{~h}$ under vacuum at $80{ }^{\circ} \mathrm{C}$ before it was weighed (mass $m_{\text {sox }}$ ) to calculate the gel fraction and the sol fraction.

$$
\phi_{\mathrm{gel}}=m_{\mathrm{sox}} / m_{0} ; \quad \phi_{\mathrm{sol}}=1-\phi_{\mathrm{gel}}
$$

Sample Preparation by the CSX Process. In this CSX process, the cross-linked HDPE sample of mass $1-2 \mathrm{~g}$ was put in a highpressure vessel and heated to $175^{\circ} \mathrm{C}$. Propane was introduced into the system and then pressurized to $62 \mathrm{MPa}$. After a swelling time of $1.5 \mathrm{~h}$, the temperature was reduced to $85^{\circ} \mathrm{C}$ while maintaining the pressure and held there for $1 \mathrm{~h}$ to crystallize the polymer. The temperature was raised above the critical temperature of the swelling fluid (the critical point of propane is at $96{ }^{\circ} \mathrm{C}$ and $4.2 \mathrm{MPa}^{17}$ ), to $110{ }^{\circ} \mathrm{C}$, before the pressure was released. After propane was completely vented, the system was cooled to room temperature and the porous sample was recovered.

Compressive Modulus Measurements. The compressive moduli of the cross-linked HDPE samples before and after CSX were measured in a Rheometrics RDS-LA linear rheometer. The samples were compressed at a constant strain rate of $0.005 \mathrm{~s}^{-1}$, and the resulting compressive force was measured. Strain rate $\dot{\epsilon}$ and strain $\epsilon$ are defined with the sample height $H(t)$ :

$$
\dot{\epsilon}=\frac{\mathrm{d}(\ln H(t))}{\mathrm{d} t} ; \quad \epsilon=\ln \left(\frac{H(t)}{H_{0}}\right)
$$

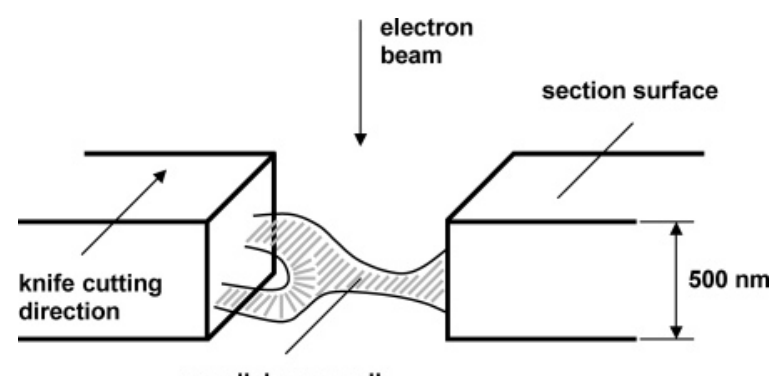

Figure 1. Illustration of a microtomed thick section of the porous HDPE-10 after CSX with an embedded single pore wall layer for TEM observation.

Stress was calculated by dividing the measured normal force by the cross-sectional area of the sample. The compressive modulus $E$ is defined as the slope of the best linear fit of the stress-strain curve. The molecular weight of strands between cross-links $M_{\mathrm{C}}$ was estimated from $E$ :

$$
M_{\mathrm{C}}=\frac{3 R T \rho \phi_{\mathrm{gel}}}{E}
$$

where $R$ is the universal gas constant, $T$ is the experimental temperature in absolute scale, and $\rho$ is the density of the polymer.

DSC Characterization. The melting behavior of HDPE-10 before and after CSX was studied by differential scanning calorimetry using a TA Instruments Q1000 DSC. The instrument was calibrated using indium as standard. Melting endotherms were obtained at a heating rate of $10^{\circ} \mathrm{C} / \mathrm{min}$ using $5 \mathrm{mg}$ of sample. The degrees of crystallinity were calculated from the heat of fusion using $293 \mathrm{~J} / \mathrm{g}$ as the heat of fusion of $100 \%$ crystalline polyethylene. ${ }^{18}$

SEM. The pore structure of the porous HDPE-10 sample after CSX was viewed under a JEOL 6320F field emission gun scanning electron microscope (FEGSEM). To expose the internal pore morphology, the porous sample was freeze-fractured in liquid nitrogen. The cracked sample was then placed on a support disk, and the fracture surface was coated with a thin layer of platinum using a Gatan 681 high-resolution ion beam coater, at a deposition rate of $1 \mathrm{~nm} / \mathrm{min}$ for $4 \mathrm{~min}$. The platinum coating thus obtained was about $4 \mathrm{~nm}$ thick, which not only effectively covered the massive surface area in the porous sample to prevent charging in the SEM but also made high-resolution imaging possible due to its fine granular structure.

TEM and ED. The microstructure of HDPE-10 before and after CSX was analyzed by transmission electron microscopy (TEM) using a JEOL $2000 \mathrm{FX}$ TEM at $200 \mathrm{kV}$ accelerating voltage. For the cross-linked HDPE sample before CSX, ultrathin sections of about $50 \mathrm{~nm}$ in thickness were cut using a Leica Ultracut cryomicrotome and a Diatome diamond knife at a knife/sample temperature of $-150{ }^{\circ} \mathrm{C}$. The sections were collected on copper grids and stained in the vapors of ruthenium tetraoxide $\left(\mathrm{RuO}_{4}\right)$ for $1 \mathrm{~h}$ before being examined in TEM. For the porous CSX processed HDPE, it would be too difficult to obtain ultrathin TEM sections using microtomy. The high sample porosity would cause thin sections to fall apart. Instead, the porous sample was microtomed at a thickness of $500 \mathrm{~nm}$, using the same temperature setting as that for the sample before $\mathrm{CSX}\left(-150^{\circ} \mathrm{C}\right)$. The sections were then stained in $\mathrm{RuO}_{4}$ vapors for $1 \mathrm{~h}$ before TEM examination. An illustration of this technique is shown in Figure 1. Although the majority of the sectioned material should be overlapped randomly oriented interconnected pore walls (depicted as thick blocks in Figure 1) that would be too thick for TEM observation, it was expected that within the thick sections occasionally there should be see-through areas (the middle region in Figure 1) where single layer of the pore wall lies approximately parallel to the section surface, thus providing a direct view of the pore wall crystal morphology in the TEM. Because only the upper and lower surfaces of the thick section were ever touched by the diamond knife during the microtoming process, the single pore wall layer observed in 
Table 1. Gel Fraction, Crystallinity, and Melting Temperatures of HDPE-10 before and after CSX

\begin{tabular}{lccccc}
\hline \multicolumn{1}{c}{ sample } & $\begin{array}{c}\text { gel } \\
\text { fraction } \\
(\%)\end{array}$ & $\begin{array}{c}\text { compressive } \\
\text { modulus } \\
(\mathrm{MPa})\end{array}$ & $\begin{array}{c}M_{\mathrm{c}} \\
(\mathrm{g} / \mathrm{mol})\end{array}$ & $\begin{array}{c}\text { crystallinity } \\
(\%)\end{array}$ & $\begin{array}{c}T_{\mathrm{m}} \\
\left({ }^{\circ} \mathrm{C}\right)\end{array}$ \\
\hline HDPE-10 before CSX & 41 & 46 & 7300 & 62 & 132 \\
HDPE-10 after CSX & & 5.1 & & 69 & 127
\end{tabular}

the TEM was effectively embedded inside the thick section due to the interconnectivity of the pore walls and therefore protected from any possible external deformation. Thus, the TEM morphology obtained for the single pore wall layer should reflect the true morphology of the pore walls in the porous CSX-processed HDPE material.

In performing electron diffraction (ED) and TEM, care was taken to limit unnecessary electron beam exposure, thus minimizing electron beam damage and producing the best possible electron diffraction patterns. This included such standard procedures as focusing on an adjacent area to the area where data was recorded, using the smallest possible spot size, limiting the spread of the beam, and turning the beam off when it was not needed. Also, the ED data were recorded on fast X-ray film (Kodak DEF5) rather than standard EM film. To calibrate the diffraction camera length, some sample grids were sputter-coated with gold, and the $\mathrm{Au}$ (111) ring was used as an internal standard.

\section{Results and Discussion}

Mechanical and Thermal Properties. Table 1 lists the gel fraction $\phi_{\text {gel }}$, the compressive modulus $E$, and the molecular weight between cross-links $M_{\mathrm{c}}$, of HDPE-10, as well as the crystallinity and the melting temperature $T_{\mathrm{m}}$ of the polymer before and after CSX. Both the gel fraction and the molecular weight between cross-links are indicators of the degree of crosslinking in a polymer sample. A higher degree of cross-linking is expressed by higher gel fraction and lower molecular weight between cross-links. A previous study on CSX-processed lowdensity polypropylene ${ }^{16}$ has found that the degree of crosslinking has a strong effect on pore size, with higher gel fraction and lower molecular weight between cross-links resulting in smaller pore size, although a quantitative relationship between the degree of cross-linking and pore size is not yet established. As shown in Table 1, the HDPE-10 sample used in this study has a moderate gel fraction of $41 \%$ and a molecular weight between cross-links of $7300 \mathrm{~g} / \mathrm{mol}$, indicating a moderate degree of cross-linking. The measured value of the average pore size in CSX-processed HDPE-10 will be presented in the next section. The compressive modulus of HDPE-10 after CSX, 5.1 $\mathrm{MPa}$, is almost an order of magnitude smaller than that of HDPE-10 before CSX, $46 \mathrm{MPa}$. This decrease in compressive modulus is not unexpected given the porous nature of the sample after CSX; however, 5.1 MPa is a relatively high compressive modulus for a porous polymeric material.

DSC melting endotherms of HDPE-10 before and after CSX are shown in Figure 2. The crystallinity of HDPE-10 increased from $62 \%$ to $69 \%$ after CSX, possibly due to the presence of the supercritical fluid which increases the mobility of the polymer chains and results in the crystallization of less perfect chains. On the other hand, the melting temperature decreased slightly from 132 to $127{ }^{\circ} \mathrm{C}$, and the melting peak became narrower after CSX, indicating thinner lamellae and better defined lamellar thickness in the porous HDPE-10. The inclusion of less perfect chains in the crystal lattice may also contribute to the decease of the melting temperature in the CSX-processed HDPE.

Pore Structure and Pore Size. Two typical SEM micrographs of HDPE-10 after CSX are shown in Figure 3, in which

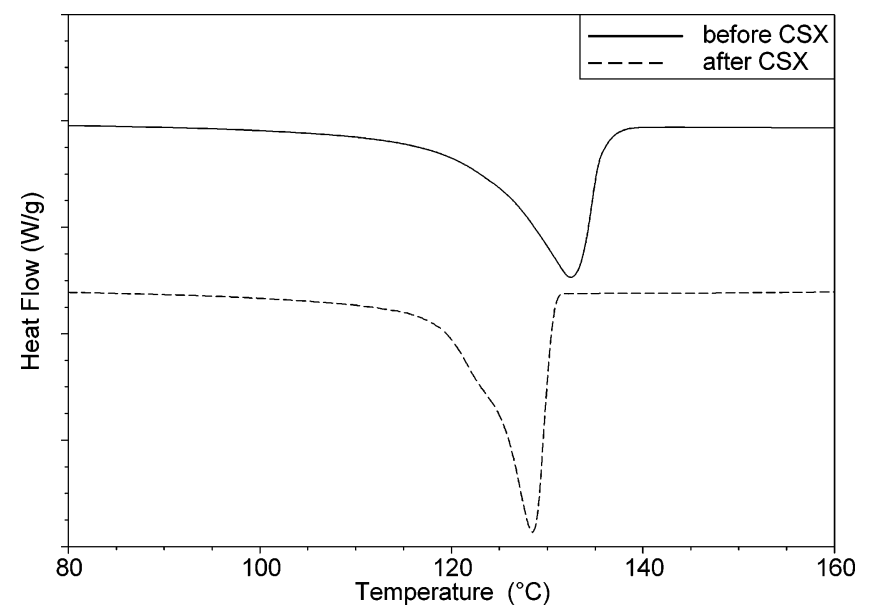

Figure 2. DSC melting endotherms for HDPE-10 before and after CSX.
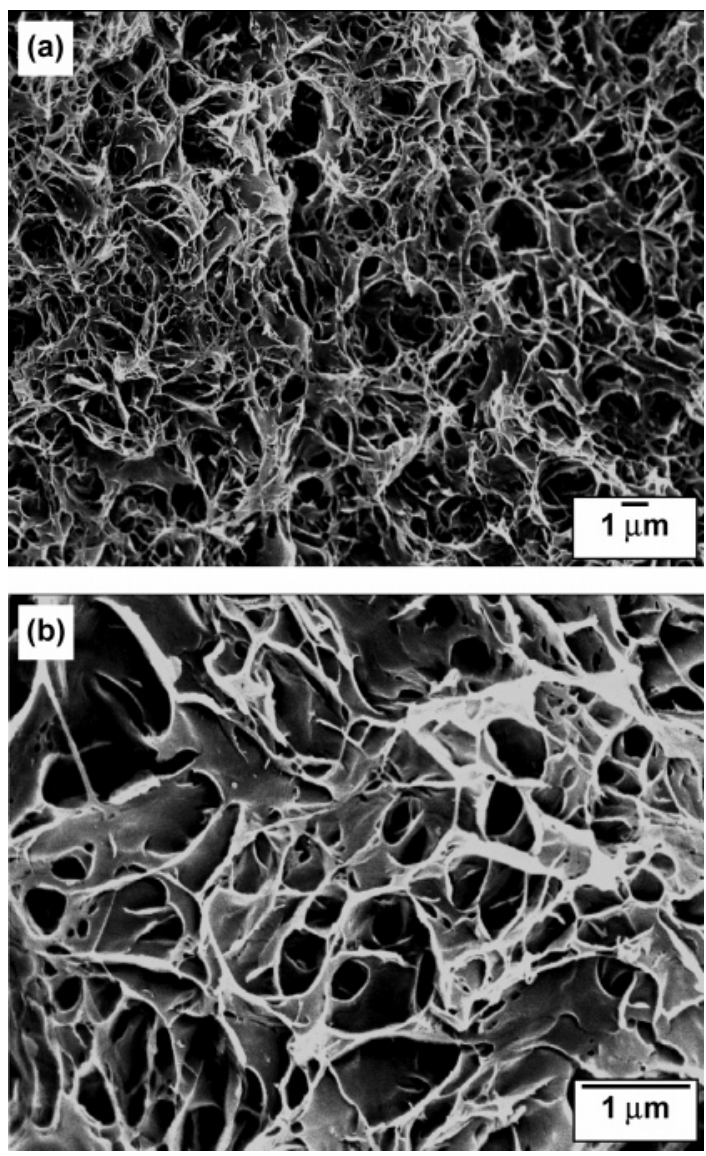

Figure 3. Pore structure of porous HDPE-10 after CSX: (a) lowmagnification SEM micrograph; (b) high-magnification SEM micrograph.

Figure 3a shows an overall pore structure at lower magnification and Figure $3 \mathrm{~b}$ shows a more localized view at higher magnification. An open-pore structure with large surface area can be seen in both micrographs. A rough measurement ${ }^{16}$ of the pore sizes from more than 20 SEM images gave an average pore size of $1-2 \mu \mathrm{m}$ for the porous HDPE-10 after CSX. High-resolution SEM images such as Figure $3 \mathrm{~b}$ reveal that smaller pores on the order of tens of nanometers are sometimes present on the pore walls. The pore walls themselves are quite thin and are on the order of $100 \mathrm{~nm}$ or thinner, as measured from the SEM images. Despite the thin walls, the pore structure is quite rigid, as demonstrated by the relatively high compressive modulus. The 

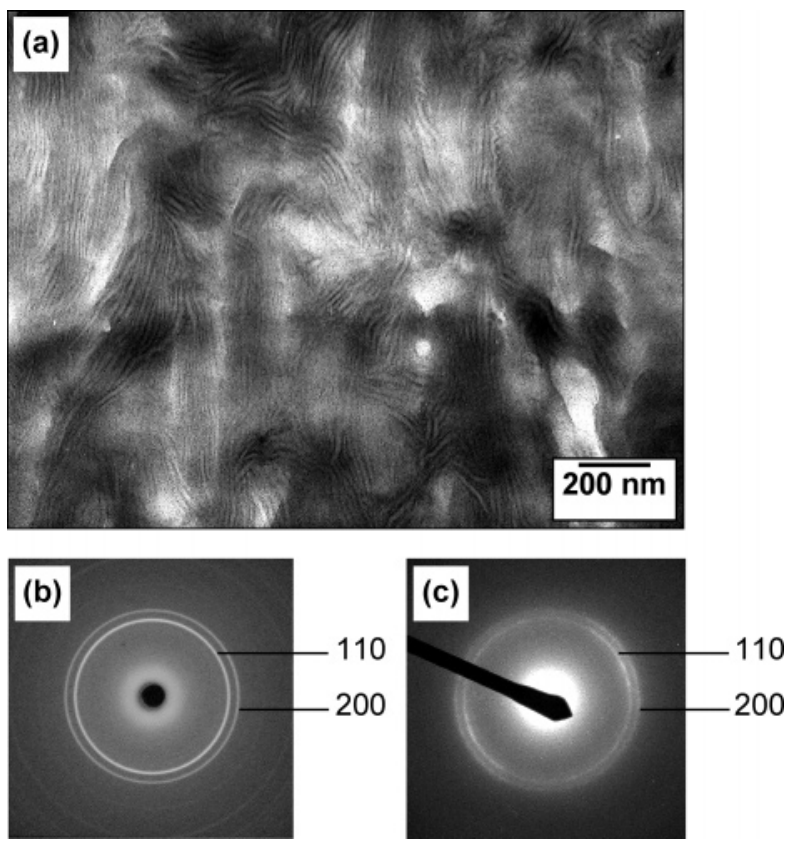

Figure 4. Bulk morphology of HDPE-10 before CSX: (a) TEM micrograph; (b) wide-angle X-ray diffraction pattern; (c) selected area electron diffraction pattern.

fact that the pore structure has survived both the fluid venting process and the cryo-fracturing process reaffirms the rigidity of the pore structure in the porous HDPE-10. The rigidity may come from (1) the presence of a continuous polymer phase and (2) the polyethylene crystallites within the pore walls.

Morphology of HDPE before CSX. To assess the degree to which the CSX process has changed the morphology of the cross-linked HDPE, TEM was performed on the HDPE-10 sample before CSX. Shown in Figure 4a is a typical TEM micrograph of the HDPE-10 morphology before CSX. The amorphous phase appears dark, and the crystalline phase appears light in the TEM micrograph due to $\mathrm{RuO}_{4}$ staining. Since the HDPE-10 sample was melt-extruded first and then e-beam crosslinked in the form of extruded sheets, its morphology is that of typical melt-crystallized polyethylene. Lamellae parallel to the plane of view, perpendicular to the plane of view, as well as those of intermediate orientations, are all clearly seen. A wideangle X-ray diffraction pattern and an electron diffraction pattern of this sample are shown in parts $b$ and $c$ of Figure 4, respectively. The two characteristic rings in both figures correspond to the 110 and 200 diffraction signals of the orthorhombic polyethylene crystal lattice. The polycrystalline rings obtained in both diffraction patterns further confirm the lack of orientational order in the cross-linked HDPE before CSX.

Morphology of HDPE after CSX. As mentioned in the Experimental Section, the porous HDPE sample after CSX were cryo-microtomed into $500 \mathrm{~nm}$ thick sections, inside of which thin areas of single pore wall were targeted for TEM examination. Figure 5a shows a representative TEM micrograph of such a see-through area in the porous HDPE-10 after CSX. In this figure, the overall morphology is that of a piece of pore wall (the middle region of the micrograph) lying almost parallel to the section surface and spanning across two thicker regions of overlapped pore walls (the upper-left and lower-right corners), which appear dark in the TEM. The geometry of this sampled area matches very well with the illustration in Figure 1. As characterized by SEM, individual pore wall thickness is on the order of $100 \mathrm{~nm}$ or less. This allows the electron beam to readily pass through the pore wall and reveal its internal structure in

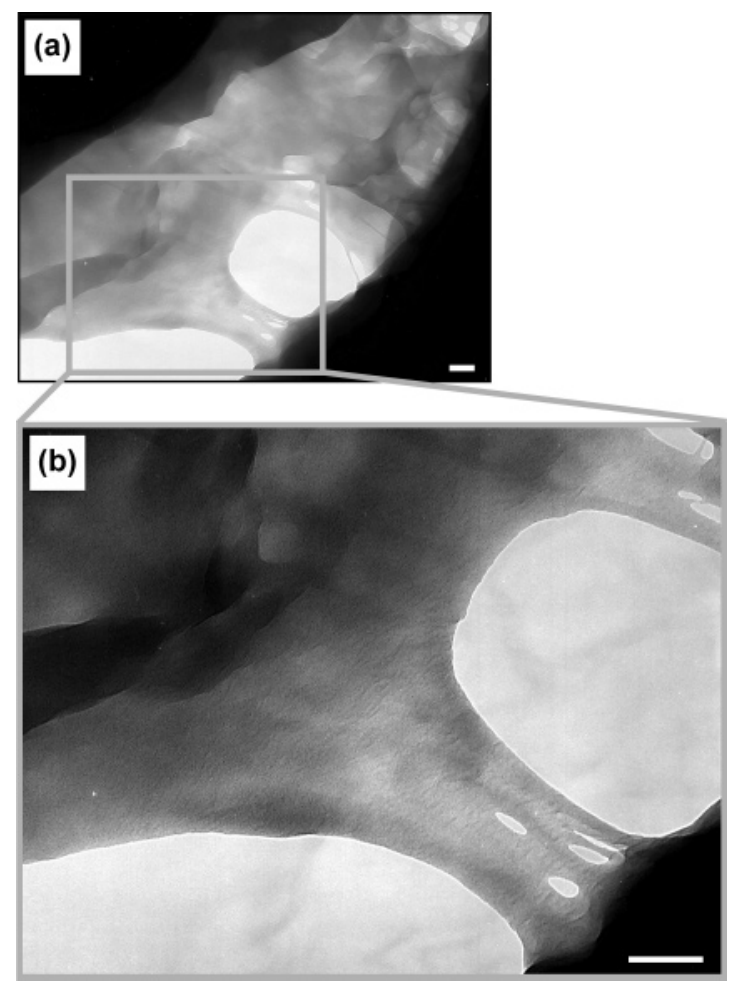

Figure 5. TEM micrographs showing pore wall morphology of porous HDPE-10 after CSX: (a) low-magnification overview of a pore wall segment spanning across two thicker overlapped regions; (b) highmagnification TEM micrograph of the boxed area in (a). Scale bar: $100 \mathrm{~nm}$.

the TEM. Conversely, the fact that TEM imaging was successful on isolated pore walls also supports our claim about the thinness of the pore wall.

In Figure 5a, the lower part of the viewable area is mostly composed of one single layer of pore wall, while the upper part is composed of two or more pore wall layers, all of which lie more or less parallel to the section surface. Despite the number of layers, two features of the pore wall are clearly visible: (1) The pore walls are indeed interconnected. (2) In addition to the large micron-sized pores, nanopores on the order of $100 \mathrm{~nm}$ or smaller are sometimes formed on the pore walls. Both features are also seen in the SEM micrographs (Figure 3).

By focusing on the boxed area (single pore wall layer) in Figure 5a at higher magnification, the TEM micrograph in Figure $5 \mathrm{~b}$ was obtained, which reveals more details of the crystal morphology of the pore wall. Clearly, the crystal morphology in Figure $5 b$ is very different from that of Figure 4a. There are several important features about this morphology. First, the lamellar crystals (light phase in the TEM micrograph) are oriented perpendicular, not parallel, to the plane of the pore wall surface. Second, the crystalline lamellae and the amorphous layers (dark phase in the TEM micrograph) stack on top of each other to form oriented lamellar stacks. Third, the lamellar crystals appear to be preferentially aligned perpendicular to the edges of the pore wall.

To explain this morphology, we propose a lamellar stacking model as shown in Figure 6. In this model, the crystalline lamellae are indicated by the light phase composed of folded chain stems, and the amorphous layers are drawn as dark regions in between the lamellae. The crystalline lamellae and amorphous layers alternate and stack along the chain axis (c-axis) of polyethylene, which is proposed to be parallel to the lamellar normal. As a starting point, it is assumed that the stacked 


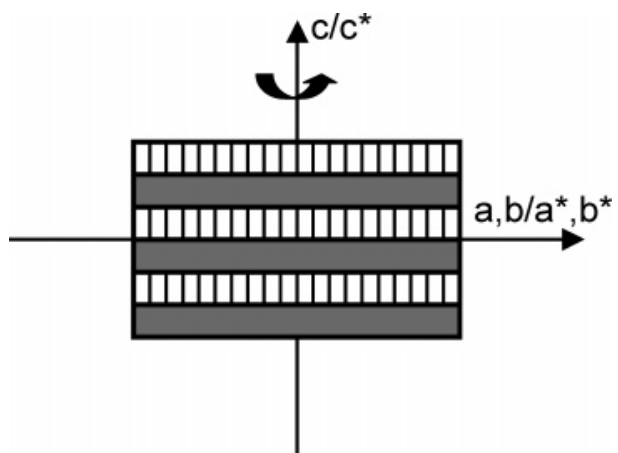

Figure 6. Proposed lamellar stacking model for the pore wall structure of porous HDPE-10 after CSX. In this model, $a, b$, and $c$ represent the crystallographic axes of the orthorhombic unit cell of polyethylene, of which $c$ is the chain axis; $a^{*}, b^{*}$, and $c^{*}$ represent the corresponding axes of polyethylene reciprocal lattice, with relationships $a\left\|a^{*}, b\right\| b^{*}$, and $c \| c^{*}$

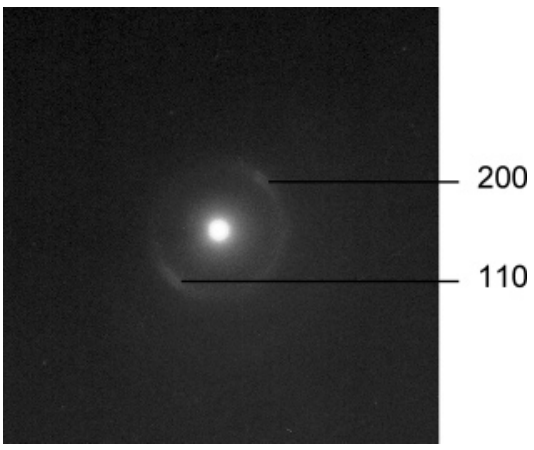

Figure 7. Selected area electron diffraction pattern from a single layer pore wall in a microtomed thick section of porous HDPE-10 after CSX.

lamellar crystals have random orientation around the chain axis, as in polyethylene fibers.

Selected area electron diffraction was performed on the microtomed pore wall sections, using a $20 \mu \mathrm{m}$ selected area aperture. This technique allows the isolation of diffraction signal from a single pore wall and thus allows evaluation of crystallite orientation within the pore wall. A representative electron diffraction pattern is shown in Figure 7, in which two pairs of arcs corresponding to 110 and 200 diffraction signals of polyethylene are seen. The observation of arcs instead of rings further indicates the oriented lamellar morphology in the pore wall. The diffraction arcs are oriented parallel to the crystalline lamellar surface, which indicates that the polyethylene chain axis ( $c$ axis) is perpendicular to the lamellar surface or parallel to the lamellar normal, as our model suggests. The observation of 110 and 200 arcs at the same time also indicates that there is a uniaxial or fiberlike orientation of the lamellae about the $c$-axis.

Origin of Lamellar Stacking and Pore Formation Mechanism in the CSX Process. The electron diffraction pattern seen in Figure 7 is quite similar to diffraction patterns of drawn polyethylene fibers. Such diffraction patterns are also observed for polyethylene crystallized in a highly stressed state. Shishkebabs from elongational flow-induced solution crystallization ${ }^{19}$ and row-nucleated structures from stress-induced melt crystallization ${ }^{20}$ both give similar diffraction patterns. In both cases, the chain axis (c-axis) is oriented in the flow (or stress) direction, and the chain-folded lamellae (kebabs in shish-kebabs and rows in row structures) are preferentially oriented perpendicular to the flow (or stress) direction or in the $a b$ plane of polyethylene crystal lattice. These characteristics match well with the lamellar stacking morphology observed in the pore walls of HDPE after CSX.

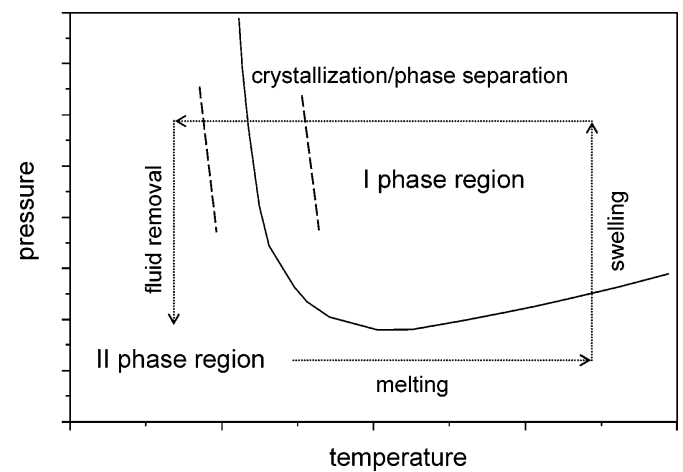

Figure 8. Phase behavior of HDPE as expected in supercritical propane. The solid line represents the temperature and pressure conditions for the phase separation of the polymer and propane. The dotted line represents the CSX process. The dashed lines represent the crystallization temperature of HDPE in the presence of supercritical propane. It is difficult to determine whether crystallization occurs prior to or after phase separation.

We have previously argued that crystallization in the CSX process is quite similar to crystallization from solution, the difference being the use of a supercritical fluid as solvent, which entails higher chain mobility. However, it is still unclear as to whether phase separation occurs due to crystallization or the gel first phase separates and then the polymer crystallizes. A phase diagram of HDPE in supercritical propane (Figure 8), similar to the one obtained for polypropylene, ${ }^{21}$ can be assumed. Thus, during the CSX process, when the temperature is reduced, HDPE and the supercritical propane should phase separate as the temperature approaches the two-phase region, shown in Figure 8. However, crystallization of the polymer might occur at a temperature above the phase-separation temperature. In such a situation, crystallization induces phase separation. Also, it is possible that the crystallization temperature of HDPE in supercritical propane is below the phase-separation temperature. In such a situation, the polymer and the swelling fluid phase separate into polymer-rich and propane-rich regions before the polymer starts to crystallize. Since it is very difficult to isolate the crystallization temperature of HDPE in supercritical propane from the phase-separation temperature, we propose possible mechanisms of crystallization and pore formation for both cases.

Crystallization Prior to Phase Separation. Because of the presence of chemical cross-links, in the highly swollen state (500-800 vol \%), although the overall stress in the polymer network is isotropic, locally the network segments can experience a high degree of stress in a specific direction. These locally oriented stretched segments have a higher crystallization temperature due to their extended-chain conformation and nucleate first as the temperature lowers in the cooling process. They nucleate as oriented fibrils along the local stretching direction upon which chain-folded lamellar crystals grow when the temperature is further lowered to their crystallization temperature. Therefore, it is likely that some of the cross-linked segments (the gel fraction) which experience highest degree of stretching crystallize first in a chain-extended fashion, and the loose chains (the sol fraction) mainly participate in subsequent lamellae crystallization transverse to those extended crystals. The rest of the network segments either crystallize in the oriented lamellae or remain noncrystalline in the amorphous regions in between the lamellar crystals. The overall result of such crystallization would be locally oriented stacked lamellae as seen in the TEM (Figure 5) micrographs.

Many such nucleating chain segments are created adjacent to each other at the onset of crystallization. The secondary 
growth (oriented lamellae) begins on each of these locally aligned nuclei. Large density fluctuations occur during this process. As chain folding and the formation of the lamellae continue, polymer chains migrate toward those nuclei creating polymer-rich and polymer-lean regions. The growth continues until the lamellae impinge. The series of impinged oriented lamellar stacks positioned next to each other create a sheet of crystalline polymer. This forms the basis of the pore wall while the polymer lean regions created during the formation of the lamellae become the pores. The pore size formed during the process is dictated by the distance between the adjacent nuclei, which in turn is determined by the cross-link density of the polymer.

During the fast growth of the lamellar crystals, the growth rate might overtake the rate of diffusion of the polymer chains. The lack of availability of the polymer creates defects in the lamellar structure, which translate into nanopores that are observed in the pore walls.

Phase Separation Followed by Crystallization. In this possible alternative scenario, as the temperature is reduced, the solvation power of supercritical propane decreases, leading to microphase separation. A thin interconnected continuous polymer-rich phase is formed which coexists with the continuous propane-rich phase. The connectivity of the polymer phase is maintained due to the presence of the cross-links, which give the structure a length scale and prevent coarsening as would be seen in classical spinodal decomposition. Thus, the cross-link density determines the size of the solvent pockets formed in between the polymer phase.

As the phase separation occurs on a microscopic scale, the molecular strands between the cross-links in the polymer network experience large amounts of strain. This strain, caused by biaxially drawing the polymer-rich microdomains into the cell walls that divide the pores, adds to the strain caused by initial swelling in the supercritical propane and results in local orientation of polymer chains. These elongated chains crystallize first and act as nucleating agents upon which the chain-folded lamellae grow transversely. Because of the 2-dimensional confinement of the crystal growth within the thin polymer phases making up the developing cell wall, the oriented lamellar growth is preferred in the plane of the pore wall, leading to the formation of high-aspect-ratio ribbonlike lamellar crystals.

It is interesting to compare results of this study to solution crystallization from supercritical solvents. Before the development of the CSX process, there have been solution crystallization studies of un-cross-linked high-density polyethylene (Winter and co-workers, unpublished) and isotactic polypropylene (Whaley et al. ${ }^{22,23}$ ), where the solvent was supercritical propane, as in this study. For both polymers, the resulting morphology differs greatly from that obtained from cross-linked polymers using CSX. Without pre-cross-linking, the porous polymer structure grown from supercritical propane has low molecular interconnectivity and relatively poor mechanical strength, suggesting that in such systems the phase separation is induced by the crystal formation. In these experiments, no polymer-rich regions were created by phase separation prior to the onset of crystallization; during crystal growth molecular chains could diffuse freely toward the growing crystal (spherulite). As a consequence, depletion regions are generated throughout the solution, resulting in semicrystalline spherulites that are poorly interconnected.

In the case of the CSX process, the polymers are pre-crosslinked. The cross-links hold the polymer together during the crystallization process. Prior phase separation is no longer necessary to create an interconnected polymer network in the
CSX-processed porous polymer. Therefore, the first mechanism, crystallization prior to phase separation, is a likely mechanism based on results from un-cross-linked polymers. On the other hand, the cross-linking may have an effect on lowering the crystallization temperature of the polymer to below its phaseseparation temperature in the supercritical fluid. If this is the case, then the second mechanism, phase separation followed by crystallization, will be a likely mechanism.

On the basis of the current data, we cannot at this time distinguish between these two possible crystallization and pore formation mechanisms. However, independent of the pore formation mechanism, we can affirm that there is no preferential direction for the lamellar stack orientation because the origin of the initial alignment of the chains is swelling, which is isotropic in macroscopic scale. Therefore, adjacent lamellar stacks may have different orientations, as observed in the TEM micrographs (Figure 5). However, the nucleating chain segments that form the backbone of the stacked lamellar structure are covalently connected because of the cross-linking. This interconnectivity of the crystalline structure provides structural integrity and significant mechanical strength to the pore walls which is extremely desirable for many applications.

\section{Conclusions}

Porous HDPE material was obtained through crystallization from swollen cross-linked polyethylene gels $\left(\mathrm{CSX}\right.$ process $\left.^{1}\right)$ in supercritical propane. SEM showed a micron-sized open-pore structure, large void fraction and surface area, and thin yet mechanically sustainable pore walls in the porous HDPE material. TEM revealed oriented lamellar structure in the pore walls, with ribbonlike lamellar crystals, much different from structures found in typical bulk HDPE material as well as that of the cross-linked HDPE before CSX processing. Electron diffraction determined the chain axis orientation as well as the random rotation of lamellar crystals in the lamellar stacks. On the basis of this oriented lamellar structure, two plausible scenarios for crystallization and pore formation in the CSX process are suggested, one favoring crystallization prior to phase separation and the other favoring phase separation prior to crystallization. It would be difficult to prove one or the other due to the possible depression of polyethylene crystallization temperature in supercritical propane.

Acknowledgment. We acknowledge support from the U.S. Army Research Laboratory-Polymer Materials Center of Excellence and the National Science Foundation (CTS 0107156), the central facilities of the NSF-supported Material Research Science and Engineering Center (MRSEC) at the University of Massachusetts Amherst, and the W.M. Keck Electron Microscopy Laboratory.

\section{References and Notes}

(1) Winter, H. H.; Gappert, G.; Ito, H. Macromolecules 2002, 35, 33253327.

(2) Pinnau, I. In Encyclopedia of Separation Science; Wilson, I. D., Ed.; Academic Press: San Diego, 2000; Vol. 4, pp 1755-1764.

(3) Mark, H. F.; Bikales, N. M.; Overberger, C. G.; Menges, G. In Encyclopedia of Polymer Science and Engineering; Mark, H. F., Ed.; John Wiley \& Sons: New York, 1990; Vol. 9, p 509.

(4) Paul, D.; Peinemann, K.-V. In Polymeric Materials Encyclopedia; Salamone, J. C., Ed.; CRC Press: Boca Raton, FL, 1996; Vol. 6, pp 4074-4082.

(5) Chiang, C.-Y.; Lloyd, D. R. J. Porous Mater. 1996, 2, 273-285.

(6) Suh, J.-K. F.; Matthew, H. W. T. Biomaterials 2000, 21, 2589-2598.

(7) Glicklis, R.; Shapiro, L.; Agbaria, R.; Merchuk, J. C.; Cohen, S. Biotechnol. Bioeng. 2000, 67, 344-353. 
(8) Ma, T.; Li, Y.; Yang, S.-T.; Kniss, D. A. Biotechnol. Bioeng. 2000, 70, 606-618

(9) Ma, P. X.; Choi, J.-W. Tissue Eng. 2001, 7, 23-33.

(10) Stachowiak, A. N.; Bershteyn, A.; Tzatzalos, E.; Irvine, D. J. Adv. Mater. 2005, 17, 399-403.

(11) Gibson, L. J.; Ashby, M. F. Cellular Solids: Structure and Properties; Cambridge University Press: New York, 1997.

(12) Saunders, J.; Hansen, R. In Plastic Foams; Frisch, K., Saunders, J., Eds.; Dekker: New York, 1972; Part I, pp 23-108.

(13) Shastri, V. P.; Martin, I.; Langer, R. Proc. Natl. Acad. Sci. U.S.A. 2000, 97, 1970-1975.

(14) Li, W.-J.; Laurencin, C. T.; Caterson, E. J.; Tuan, R. S.; Ko, F. K. J. Biomed. Mater. Res. 2002, 60, 613-621.

(15) Sakai, Y.; Otsuka, M.; Hanada, S.; Nishiyama, Y.; Konishi, Y.; Yamashita, A. Mater. Sci. Eng. C 2004, 24, 379-386.

(16) Nandi, S.; Winter, H. H.; Fritz, H. G. Polymer 2004, 45, 4819-4827.
(17) Tester, J. W.; Modell, M. Thermodynamics and Its Applications, 3rd ed.; Prentice Hall: Englewood Cliffs, NJ, 1983; Appendix G.

(18) Wunderlich, B. Macromolecular Physics; Academic Press: New York, 1980; Vol. 3.

(19) Pennings, A. J.; Kiel, A. M. Kolloid-Z. 1965, 205, 160-162.

(20) Keller, A.; Machin, M. J. J. Macromol. Sci., Phys. 1967, B1, 41-91.

(21) Whaley, P. D.; Winter, H. H.; Ehrlich, P. Macromolecules 1997, 30 , 4887-4890.

(22) Whaley, P. D.; Kulkarni, S.; Winter, H. H.; Stein, R. S.; Ehrlich, P. Polym. Mater. Sci. Eng. 1995, 73, 404-405.

(23) Whaley, P. D.; Kulkarni, S.; Ehrlich, P.; Stein, R. S.; Winter, H. H.; Conner, W. C.; Beaucage, G. J. Polym. Sci., Polym. Phys. 1998, 36, 617-627.

MA051093D 